\title{
Alianza para el conocimiento: Universidad y organización cooperativa
}

Roxana Sánchez Bozaำ

Sumario: 1. Nuestro punto de partida: la educacion en general,-2. El quinto principio: educacion entrenamiento e informacion,-3. Papel de las universidades en el desarrollo del quinto principio cooperativo: la formacion cooperativa, - 4 . La red universitaria de las americas en estudios cooperativos y asociativismo,- - 5 . Programa de maestria en administracion de empresas con enfasis en organizaciones cooperativas, universidad de costa rica,-6. Convenio con la universidad estatal a distancia y entes publicos y cooperativos para desarrollar un programa de licenciatura en administracion en empresas con enfasis en cooperativas y asociativas, -7 . Convenios de la universidad nacional con el movimiento cooperativo,-8. Contribucion de las universidades privadas al desarrollo del quinto principio cooperativo. La labor de la fundepos-alma mater,-9. Programas interuniversitarios relacionados con las cooperativas. Más alla del cooperativismo: la economia social. Red euro-latinoamericana de estudios en economia social y coooperativa, (rulescoop) del proyecto alfa, union europea en latinoamerica, conclusiones

\section{Nuestro punto de partida: la educación en general}

En los finales del Siglo xx encontramos varios planteamientos de educadores que fueron designados como partícipes de interesantes grupos de análisis del estado de situación de la educación mundial, para determinar los retos, desafíos y posibles soluciones para su desarrollo positivo al ser humano.

Lo interesante de sus resultados fue que posaron su mirada en dos de los principales aspectos que han caracterizado la educación cooperativa: en cuanto a las políticas de educación consideraron que las mis-

\footnotetext{
${ }^{1}$ Abogada y Notaria PúBlica Presidenta Asociación costarricense Derecho Cooperativo.
} 
mas deben tender a la educación como un proceso permanente de enriquecimiento de los conocimientos, de la capacidad técnica, pero también, y quizás sobretodo, como una estructuración privilegiada de la persona y de las relaciones entre individuos, entre grupos y entre naciones. $^{2}$

Asimismo, quienes integraron la Comisión que realizó el estudio antes mencionado, y por mandato de la UNESCO, consideraron como función central de la misma y en función de la esperanza de un mundo mejor, capaz de respetar los derechos del hombre y la mujer, practicar el entendimiento mutuo y hacer del progreso del conocimiento un instrumento de promoción del género humano y no de discriminación. ${ }^{3}$

2 Resumen del Informe de la Comision Internacional sobre la Educacion para el siglo XXI, a la UNESCO, presidida por Jacques DeLORS, p. 10. en La Educacion Encierra un Tesoro. Informe a la UNESCO de la Comisión Internacional sobre la Educación para el Siglo XXI. Compendio. Ediciones UNESCO

${ }^{3}$ Aspectos de nuestro interés para considera que el cooperativista así como el docente se mueve en un mundo que cada vez se ensancha más y obliga a abarcar más conocimiento, pero también a ser más tolerante, solidario, cooperador y tener un mayor conocimiento de sí mismo, a la vez que del prójimo, y de la comunidad nacional e internacional o planetaria en que cada uno de nosotros nos desarrollamos, La COMISION presidida por Delors ha identificado varias tensiones que el ser humano debe enfrentar y buscar soluciones desde sí mismo y con las herramientas que la educación a que ha accedido le ofrece. Mencionamos algunas de esas tensiones para tenerlas en cuenta en este planteamiento inicial y general:

"Las tensiones que han de superarse

A este fin conviene afrontar, para superarlas mejor, las principales tensiones que, sin ser nuevas, están en el centro de la problemática del siglo XXI.

La tensión entre lo mundial y lo local: convertirse poco a poco en ciudadano del mundo sin perder sus raíces y participando activamente en la vida de la nación y las comunidades de base.

La tensión entre el largo plazo y el corto plazo, tensión eterna pero alimentada actualmente por un predominio de lo efímero y de la instantaneidad, en un contexto en que la plétora de informaciones y emociones fugaces conduce incesantemente a una concentración en los problemas inmediatos. Las opiniones piden respuestas y soluciones rápidas, mientras que muchos de los problemas encontrados necesitan una estrategia paciente, concertada y negociada de reforma. Tal es precisamente el caso de las políticas educativas.

La tensión entre la indispensable competencia y la preocupación por la igualdad de oportunidades. Cuestión clásica, planteada desde comienzo de siglo a las políticas económicas y sociales y a las políticas educativas; cuestión resuelta a veces pero nunca en forma duradera. Hoy, la Comisión corre el riesgo de afirmar que la presión de la competencia hace olvidar a muchos directivos la misión de dar a cada ser humano los medios de aprovechar todas sus oportunidades. Esta constatación nos ha conducido, en el campo que abarca este informe, a retomar y actualizar el de educación durante toda la vida, para conciliar la competencia que estimula, la cooperación que fortalece y la solidaridad que une. 
Por su parte, Edgar Morin consideró que existen SIETE SABERES DEL CONOCIMIENTO que deben ser tomados en cuenta en cualquier proceso educativo ${ }^{4}$, del futuro, en cualquier sociedad y en cualquier cultura sin excepción alguna ni rechazo según los usos y las reglas propias de cada sociedad y de cada cultura, que por lo tanto son aplicables al Cooperativismo, en nuestra opinión, como movimiento mundial que se aplica a múltiples grupos, culturas en todos los continentes, y que tienen diversos desarrollos económicos, políticos y sociales.

El planteamiento de este autor, nos llama la atención porque no debemos olvidarlo cuando desarrollamos el quinto principio de la doctrina cooperativista y a la vez, nos centra en el ser humano, sus características como tal y su inserción en su comunidad e integración con resto del mundo, o sea su naturaleza planetaria.

Luego de enunciar los saberes elegidos por Morin, en forma resumida, nos podemos preguntar si deben formar parte de los programas universitarios que se ofrecen al Cooperativismo, o si forman parte del bagaje educacional que se aporta a la educación universitaria, que generalmente se enfatiza en la formación y el entrenamiento. Veamos por ahora esos saberes:

1. Las cegueras del conocimiento: el error y la ilusión

Morín nos indica que es necesario introducir y desarrollar en la educación el estudio de las características cerebrales, mentales y culturales del conocimiento humano, de sus procesos y modalidades, de las disposiciones tanto síquicas como culturales que permiten arriesgar el error o la ilusión.

\section{Los principios de un conocimiento pertinente}

Es necesario desarrollar la aptitud natural de la inteligencia humana para ubicar todas sus informaciones en un contexto y en un conjunto. Es necesario enseñar los métodos que permiten aprehender las relaciones mutuas y las influencias recíprocas entre las partes y el todo en un mundo complejo.

Por tanto, será necesario escoger, en una clara estrategia de reforma, pero a condición de preservar los elementos esenciales de una educación básica que enseñe a vivir mejor mediante el conocimiento, la Experimentación y la formación de una cultura personal.

Por último, la tensión entre lo espiritual y lo material, que también es una constatación eterna. E1 mundo, frecuentemente sin sentirlo o expresarlo, tiene sed de ideal y de valores que vamos a llamar morales para no ofender a nadie»

4 Publicado por la UNESCO, en octubre de 1999 por la Organización de las Naciones Unidas para la Educación, la Ciencia y la Cultura -7 place de Fontenoy - 75352 París 07 SP - Francia. 1999 


\section{Enseñar la condición humana}

El ser humano es a la vez físico, biológico, psíquico, cultural, social, histórico. Es esta unidad compleja de la naturaleza humana la que está completamente desintegrada en la educación a través de las disciplinas y que imposibilita aprender lo que significa ser humano. Hay que restaurarla de tal manera que cada uno desde donde esté tome conocimiento y conciencia al mismo tiempo de su identidad compleja y de su identidad común a todos los demás humanos.

\section{Enseñar la identidad terrenal}

En lo sucesivo, el destino planetario del género humano será otra realidad fundamental ignorada por la educación. El conocimiento de los desarrollos de la era planetaria que van a incrementarse en el siglo XXI y el reconocimiento de la identidad terrenal que será cada vez más indispensable para cada uno y para todos deben convertirse en uno de los mayores objetos de la educación.

\section{Enfrentar las incertidumbres}

Se tendrían que enseñar principios de estrategia que permitan afrontar los riesgos, lo inesperado, lo incierto, y modificar su desarrollo en virtud de las informaciones adquiridas en el camino. Es necesario aprender a navegar en un océano de incertidumbres a través de archipiélagos de certeza.

\section{Enseñar la comprensión}

La comprensión es al mismo tiempo medio y fin de la comunicación humana. Ahora bien, la educación para la comprensión está ausente de nuestras enseñanzas. El planeta necesita comprensiones mutuas en todos los sentidos. Teniendo en cuenta la importancia de la educación para la comprensión en todos los niveles educativos y en todas las edades, el desarrollo de la comprensión necesita una reforma de las mentalidades. Tal debe ser la tarea para la educación del futuro.

\section{La ética del género humano}

La ética no se podría enseñar con lecciones de moral. Ella debe formarse en las mentes a partir de la conciencia de que el humano es al mismo tiempo individuo, parte de una sociedad, parte de una especie. Llevamos en cada uno de nosotros esta triple realidad. De igual manera, todo desarrollo verdaderamente humano debe comprender el desarrollo conjunto de las autonomías individuales, de las participaciones comunitarias y la conciencia de pertenecer a la especie humana. 
De allí, se esbozan las dos grandes finalidades ético-políticas del nuevo milenio: establecer una relación de control mutuo entre la sociedad y los individuos por medio de la democracia y concebir la Humanidad como comunidad planetaria. La educación debe no sólo contribuir a una toma de conciencia de nuestra Tierra-Patria, sino también permitir que esta conciencia se traduzca en la voluntad de realizar la ciudadanía terrenal.

Como respuesta a nuestra interrogante sobre la ubicación de los saberes elegidos por Morin, consideramos que por dar la real importancia al ser humano y a su relación con el prójimo en un mundo interior, así como planetario, y ser parte de la propia naturaleza de ese ser, en cualquier etapa de la educación, sea en la inicial, en la formativa o en el entrenamiento, ninguno de esos saberes pueden dejarse de lado, pues son interdependientes entre sí y por lo tanto necesarios para formar un ser humano de gran valía.

En el desarrollo de la parte correspondiente a este ensayo a la relación de Universidad y Cooperativismo, nos hemos encontrado ofertas académicas dirigidas esencialmente a la formación y entrenamiento, que pertenecen a las Universidades organizadas en torno a la Administración de empresas con énfasis en finanzas, banca y otros aspectos.

Otras universidades comprometidas con la ideología y filosofía cooperativista, incluyen en sus programas cursos que demuestran un mayor ligamen con el ser cooperativista y como apoyo al desarrollo de las organizaciones cooperativas y también de las asociativas.

En la región Centroamericana y del Caribe, funcionan básicamente universidades en Costa Rica con oferta académica de programas dirigidos al Cooperativismo del país y la región. Aparte de dos excelentes programas interuniversitarios impulsados por UNIRED y el programa ALFA.

\section{El quinto principio: educación entrenamiento e información}

Si consideramos el papel de las Universidades en el desarrollo del Cooperativismo, tenemos que buscar su vínculo directo con alguno de los principios que informan la Identidad Cooperativa, concepto ampliamente desarrollado a partir del año 1995, con la Declaración de Identidad Cooperativa, en Manchester Inglaterra. Eso sí nos preguntamos, ¿en qué parte del desarrollo de ese Principio pueden ingresar las universidades?

Cuando las personas acceden a los estudios universitarios, ya han pasado por una etapa básica en su formación personal, es en los pri- 
meros diez o doce años que las personas, en general, adquieren la formación básica en cuanto al conocimiento de su ser, de los valores morales y las herramientas básicas en cuanto a escritura, comprensión de su entorno y la dirección que puede tomar su vida o que debe elegir, o bien, forzosamente se ve presionado a ingresar en un determinado trabajo, a realizar determinado oficio o estudiar una profesión que se supone le dará muchos frutos económicos en su vida.

¿Cómo se ve actualmente la Educacion Cooperativa?

Encontramos la respuesta básicamente a través del quinto principio cooperativo que debe ser comprendido en forma interdependiente con el resto de los principios cooperativos y son medio de la práctica de los valores cooperativos, y reza

Quinto Principio: Educación, Entrenamiento e Información. Las cooperativas brindan educación y entrenamiento a sus socios y socias, a sus dirigentes electos, gerentes y empleados, de tal forma que contribuyan eficazmente al desarrollo de sus cooperativas. Las cooperativas informan al público en general, particularmente a los jóvenes y a los creadores de opinión, acerca de la naturaleza y beneficios del cooperativismo ${ }^{5}$.

Es indiscutible que la educación es el vehículo capaz de propiciar transformaciones sociales. El ser humano es el protagonista y motor principal del desarrollo, por lo que la tarea de la educación es formarlo íntegramente, capacitándolo para enfrentarse a un mundo cada día más complejo y en constante evolución.

La educación cooperativa tiene entre sus tareas dar a conocer qué es una cooperativa y promover sus valores y principios tanto en su medio como en la población en general. Si ella logra ser asimilada e integrada dentro de las cooperativas, estará comenzando un proceso de fortalecimiento y aprendizaje entre sus miembros que contribuirá a elevar su nivel de vida económico y social.

La educación cooperativa comprende el desarrollo, la transmisión y adquisición de nuevos conocimientos y habilidades por los miembros de una cooperativa con el fin de que cada uno pueda ejercer mejor su rol dentro de su unidad productiva. Es una educación que promueve en los cooperativistas los valores de solidaridad, cooperación, autonomía, democracia, colectivismo e igualdad.

5 Tomado de la página Web de la $\mathrm{ACl}$ : Documento Informativo N. ${ }^{\circ}$ EHO/101/95 del 17 de noviembre de 1995) (Publicado en «Mundo Cooperativo», N. ${ }^{\circ} 11$, mayo de 1997, Pág. 24, bajado el 20 de junio de 2008. 
Los valores y como hemos indicado, el resto de los principios cooperativos complementan la IDENTIDAD COOPERATIVA, los valores establecidos en encuentro del 3 de septiembre de 1995 de la Alianza Cooperativa Internacional, en el Congreso realizado en Manchester, Reino Unido son

\section{Valores}

Las cooperativas se basan en los valores de ayuda mutua, responsabilidad, democracia, igualdad, equidad y solidaridad. Siguiendo la tradición de sus fundadores, los miembros de las cooperativas creen en los valores éticos de honestidad, transparencia, responsabilidad social y preocupación por los demás.

De importancia para este ensayo consideramos importante agregar en forma especial, otros principios como son

Sexto principio: Cooperación entre Cooperativas. Las cooperativas sirven a sus socios más eficazmente y fortalecen el movimiento cooperativo, trabajando de manera conjunta por medio de estructuras locales, nacionales, regionales e internacionales.

Séptimo Principio: Compromiso con la Comunidad. La cooperativa trabaja para el desarrollo sostenible de su comunidad por medio de políticas aceptadas por sus socios.

Porque es la relación de las cooperativas con otras cooperativas así como con otros entes, vinculación que puede adquirir la forma de alianza estratégica para el desarrollo del quinto principio, y que ayuda, colabora con la función social que debe cumplir el Cooperativismo dentro de la comunidad, nuevo principio introducido en Manchester, que lleva al cooperativismo, a pensar más allá del adoctrinamiento en los componentes de la Identidad cooperativa, e incorporar a otras estructuras organizacionales en el desarrollo de su vida diaria y de sus miembros.

La unión del quinto, sexto y sétimo principio nos permite determinar en forma más concreta el rol que juega cada uno y su vinculación con el aspecto del desarrollo del ser humano y la organización cooperativa como herramienta de su evolución económico-social

— Regla de oro del cooperativismo: Educación, formación e información.

La educación cooperativa consiste en la adquisición de hábito de pensar y juzgar de acuerdo con los principios y el ideal cooperativo. Respecto de la extensión de este principio hay que distinguir: 
- Destinatarios: La educación debe ir dirigida tanto a los socios y las socias., dirigentes, a los empleados y las empleadas de la cooperativa, como al pueblo en general.

- Contenido: No solo debe referirse a los principios y técnicas cooperativas sino también trascender a la instrucción pública en términos generales.

- Cooperación entre cooperativas o principio de integración cooperativa. Tiene varios aspectos:

- Carácter societario-empresarial con la finalidad de potenciar la acción empresarial y el objeto social de 2 o más cooperativas con intereses homogéneos.

- Carácter asociativo más amplio: Al servicio de los intereses generales de las cooperativas y del cooperativismo federaciones y confederaciones cooperativas).

- Carácter solidario de integración con entes externos a las cooperativas: cuando se aprovecha la unión con entes con objetivos sociales análogos a los objetivos de las cooperativas, o se aprovechan uniones empresariales para conseguir el objetivo económico social de las cooperativas, siempre y cuando no lleven a desnaturalizar su Identidad Cooperativa.

- Interés por la comunidad: Las cooperativas deben trabajar para conseguir el desarrollo sostenible de la propia entidad y el entorno.

\section{Papel de las universidades en el desarrollo del quinto principio cooperativo: la formacion cooperativa}

Como dijimos queda en la escuela y los colegios el papel de ofrecer educación al ser humano, ya en el campo universitario, las personas acceden al conocimiento más elaborado, aquel conocimiento que será su herramienta especializada de trabajo y que le ofrecerá mejores oportunidades de inserción en el mercado de trabajo.

Podríamos partir de la interrogante siguiente ¿Las universidades están obligadas a ofrecer doctrina cooperativa dentro de sus curricula, cuando ofrecen formación a los cooperativistas, o el desarrollo de tal materia debe quedar solo en las manos del cooperativismo de cada país, o en los programas desarrollados por entes regionales, como en el caso de Centroamérica y el Caribe, por la Confederación de cooperativas de Centroamérica y el Caribe, o mundiales, y por tanto con incidencia en esa región, como es la Alianza Cooperativa Internacional? 
La respuesta no es fácil, en el tanto que si asumimos el planteamiento de la Comisión Delors que considera que la educación es para siempre, la ubicación del aprendizaje de la doctrina cooperativa, que comprende Principios y valores cooperativos, como parte de la Identidad Cooperativa, cada vez que se adquieren nuevos conceptos o se aprenden y aprehenden nuevas herramientas de desarrollo profesional, está presente la influencia y la obligación de los cooperativistas que acceden a estudios universitarios, de realizar un constante cotejo con esa doctrina con el fin de evitar desviaciones e impedir incrustaciones en el modo de ser cooperativo.

El Rector de la Universidad de Sherbrooke, Canadá, Bruno-Marie Béchard expresa muy bien este planteamiento con las siguientes palabras:

«Para comenzar, es de vital importancia formar personas solidarias con una posición ética, que hagan de la cooperación un proyecto de vida consciente ya que los cooperativistas constituyen el capital social necesario para transformar la sociedad». ${ }^{6}$

Bueno no podemos intuir del análisis de los programas universitarios ofrecidos a los movimientos cooperativos, si hay la toma de posición de formar personas solidarias, en el tanto que la mayoría de las carreras universitarias están dirigidas a desarrollar aptitudes, conocimientos, destrezas en el desarrollo de organizaciones empresariales más que organizaciones cooperativas imbuidas en el concepto de Identidad Cooperativa.

Lo anterior nos lleva a considerar la posición de dos universidades fuera de la región centroamericana y caribeña, con una extensa tradición de formación de cooperativistas, de profesionales o técnicos a su servicio, que se han autoproclamado y han hecho una forma de vida universitaria, la Identidad Cooperativa, con carreras universitarias y con programas de extensión universitaria que benefician el desarrollo de un ser humano solidario y por lo tanto identificado con la ideología, principios y valores cooperativos. Esas universidades son: la Universidad de Deusto y la Universidad de Sherbrooke, en continentes diferentes: Europa y América, pero con intereses similares con su verdadera identificación con el Cooperativismo como doctrina y forma de vida de los humanos y las humanas que se forman en sus campus.

La Universidad de Deusto con una larga tradición en su identificación con el Cooperativismo del país vasco, y del mundo sobre todo

${ }^{6}$ Bruno-Marie Béchard, Rector de la Université de Sherbrooke (Canadá). La cooperación como orden mundial, discurso con motivo del X Seminario Internacional de la Red universitaria de las Américas en estudios cooperativos y asociativismo (uniRcoop). Copán Ruinas, 2 de noviembre de 2006. p. 4. 
europeo, ha colaborado en el impulso de estudios cooperativos, tesis de doctorados en Empresariales y en Derecho, ha sido incubadora de empresas de profesionales cooperativistas, cuenta con un Instituto de estudios Cooperativos y es seno de la Asociación internacional de Derecho Cooperativo con su excelente publicación del Boletín de esa agrupación. Y dejamos a nuestros colegas españoles el desarrollo de la interesante labor de esta Universidad.

La Universidad de Sheerbroke, nacida en Québec, Canadá también está comprometida en forma transversal con los estudios cooperativos, con acciones como el desarrollo del Instituto de investigación cooperativa, IRECUS, carreras especializadas en aspectos empresariales cooperativos, ha implementado múltiples convenios con otras universidades de América, entre ellas la Universidad de Costa Rica, con el desarrollo de la Maestría de administración de empresas cooperativas de la Facultad de Economía y múltiples proyectos en la región centroamericana y del caribe, como es el apoyo de la UniRed.

Béchard, Rector de la Université de Sherbrooke (Canadá) expresa el compromiso de la Universidad considerando los contenidos que realmente deben incorporarse en los programas de formación de cooperativistas y auxiliares de cooperativas y dice:

«El desarrollo del cooperativismo requiere específicamente la formación de cooperativistas, la concepción de estructuras organizacionales colectivas, la ínter cooperación y la unión con los sectores privado y público para formar una tríada. Es por eso que desde 1976, la Université de Sherbrooke ha hecho del cooperativismo una de sus "orientaciones importantes» implicando al conjunto de la institución. Al crear el Instituto de investigación y de educación para las cooperativas y las mutuales (IRECUS), Sherbrooke consagró el carácter multidisciplinario del sector COOP e intensificó su contribución al progreso del movimiento, tanto en Québec como internacionalmente. ${ }^{7}$

\section{La red universitaria de las americas en estudios cooperativos y asociativismo}

La red nació en la década de los 90 del Siglo xx, cuando investigadores especializados en el fenómeno cooperativo y asociativo de Amé-

7 Bruno-MARIE Béchard, Rector de la Université de Sherbrooke (Canadá). La Cooperación Como Orden Mundial, discurso con motivo del X Seminario Internacional de la Red universitaria de las Américas en estudios cooperativos y asociativismo (uniRcoop). Copán Ruinas, 2 de noviembre de 2006. p.3 
rica de todos los rincones de las Américas se agruparon en una red única, su objetivo esencial fue convertirse en un lugar privilegiado de reflexión y difusión sobre el paradigma cooperativo y contribuir al desarrollo del cooperativismo ofreciendo soluciones a los desafíos del nuevo milenio.

La Red uniRcoop está basada en un proyecto de cooperación universitaria denominado "Modelos de ínter cooperación para las Américas», financiado en el marco del Programa de asociaciones universitarias en cooperación y desarrollo (PPUCD), aspecto 1, de la Agencia Canadiense de Desarrollo Internacional (ACDI). Con una duración de cinco años, el proyecto comenzó en abril de 2002 y su financiamiento llegará a término en junio de 2007 . El proyecto se propone responder a los desafíos del desarrollo, contribuyendo a la educación y desarrollo de las cooperativas, a través del logro de un mejor ínter cooperación en las Américas.

Específicamente, el proyecto «Modelos de ínter cooperación para las Américas» cuenta con cinco universidades partenaires y catorce universidades asociadas distribuidas en quince países de las Américas. A través del proyecto, los cinco partenaires principales, es decir, la Universidad de Costa Rica (Costa Rica), la Universidade Federal Rural de Pernambuco (Brasil), la Universidad de Chile (Chile), la Pontificia Universidad Javeriana (Colombia) y la Universidad de Sherbrooke (Canadá), contribuyen a habilitar a las poblaciones locales de América a través de sus cooperativas, a cooperar con mayor eficacia económica, social y medioambiental, garantizando así una mejora de las condiciones de vida.

Lugar de convergencia de los saberes cooperativos

— Desarrollo de modelos de ínter cooperación

- Enseñanza conjunta

— Formación sobre medida

- Servicios a la colectividad adaptados

- Revista científica UniRcoop

- Seminarios internacionales

Estructura de la red uniRcoop

Todas las universidades miembros de la Red uniRcoop cuentan con una especialización en cooperativismo y/o en el fenómeno asociativo en el seno mismo de la estructura universitaria, ya sea a través de un 
programa universitario, un instituto o una unidad de investigación. La Red se divide en cinco regiones y las universidades miembro de cada una de las regiones son las siguientes:

América del Norte y el Caribe, América Central, Cono Sur parte Sur, Cono Sur parte Norte

Las cinco universidades partenaires del proyecto constituyen el Comité de Dirección de la Red uniRcoop. Este comité tiene, por una parte, la tarea de planificar, controlar y evaluar los resultados obtenidos a partir del marco lógico del proyecto y, por otra parte, garantizar la coordinación con las universidades asociadas en la región respectiva.

\section{Actividades de la Red uniRcoop}

La red desarrolla su trabajo a través de varios instrumentos de relación y difusión de sus actividades:

- El trabajo de los Comités académicos multipaíses y tienen por objetivo realizar conjuntamente actividades de investigación aplicada o de formación para las cooperativas. El objetivo consiste en favorecer el trabajo en red, el desarrollo de modelos de ínter cooperación para finalmente transferir los conocimientos desarrollados a los cooperativistas.

- Publicación anual de una revista que reúne un conjunto de artículos escritos en varios idiomas y que en su mayoría constituyen el resultado de los trabajos de los Comités académicos.

- El Seminario Internacional uniRcoop, organizado cada año en una de las universidades miembro, es la ocasión para los investigadores, los estudiantes y los cooperativistas de la Red de intercambiar sobre sus experiencias y labores de investigación vinculadas al cooperativismo.

ACl coopera con las universidades a traves de la red

La Alianza de Cooperativas para las Americas firmó un acuerdo de cooperación con la UNIRCOOP con el objetivo de apoyar el proceso de cooperación de la Red Universitaria de las Américas en Estudios Cooperativos Asociativos a ser presentada a la Asociación de Universidades y Colegios de Canadá (AUCC) en el marco del concurso «seguimiento de los proyectos exitosos», el 7 de setiembre de 2006, Su objetivo es promover el desarrollo y la difusión de la filosofía y los valores del cooperativismo y de la economía social y solidaria mediante el uso de la Nuevas 
Tecnologías de la Información y la Comunicación, así como de la educación presencial en el continente americano.

\section{Programa de maestria en administracion de empresas con énfasis en organizaciones cooperativas, Universidad de Costa Rica}

La Maestría se desarrolló en la década de los noventa, gracias a un convenio de la Universidad de Costa Rica y la Universidad de Sherbroke, Québec, Canadá. Está dirigido a formar profesionales con un alto nivel gerencial y una gran capacidad de respuesta empresarial, capaces de participar en la gestión de las empresas cooperativas, de organismos de integración y de organizaciones nacionales e internacionales, públicas y privadas, que laboran el sector cooperativo.

Es un Programa realmente identificado con los principales retos que enfrenta el Cooperativismo y el conocimiento y la utilización de herramientas administrativas que permiten gestionar con éxito estas organizaciones y ofrece posibilidad a dirigentes, administradores y cooperativistas de Centroamérica y el Caribe de adquirir mayores conocimientos o profundizarlos mediante un programa con contenidos propios de la materia cooperativa y asociativa y sus trabajos finales de graduación tienden a ofrecer soluciones a la problemática cooperativa de la región.

Este Programa cuenta con una amplia red de alianzas que parte de la Escuela de Administración Pública, Escuela de Economía, Escuela de Administración de Negocios, Instituto de Investigaciones en Ciencias Económicas (IICE), Instituto de Investigaciones Jurídicas (IIJ), el Centro de Investigación y Capacitación en Administración Pública (CICAP) todos de la Universidad de Costa Rica así como con entes cooperativos de ulterior grado como son, Red de las Américas, Proyecto Módelos de Ínter cooperación, Red de Universidades de las Américas en Estudios de Cooperativas y Asociativismo, Agencia Canadiense para la Cooperación Internacional (ACDI), Centro Nacional de Capacitación Cooperativas (CENECOOP), Confederación de Cooperativas del Caribe y Centroamérica (CCCCA), Alianza Cooperativa Internacional (ACI).

Cabe destacar la presencia de varios cursos dirigidos a profundizar en aspectos de la vida económica y social de las cooperativas, y desarrollan los siguientes aspectos:

- Elementos principales de la gestión de cooperativas, el contexto en el que estas se desenvuelven y los retos y oportunidades del desarrollo cooperativo 
- Procesos de gestión de la organización cooperativa, tomando en cuenta su doble misión: la eficiencia en tanto unidad productiva y los objetivos humanos en tanto asociación humana.

- Conceptos e instrumentos para la planificación, ejecución y control de los procesos de gestión de la cooperativa.

- Misión social de las cooperativas.

— Desarrollo de las cooperativas como unidades productivas.

\section{Convenio con la Universidad Estatal a Distancia y entes públicos y cooperativos para desarrollar un programa de licenciatura en administración en empresas con énfasis en cooperativas y asociativas}

El 12 de febrero de 2008 la Universidad de Educación a Distancia de Costa Rica, se unió con la cúpula del movimiento cooperativo para relanzar opción universitaria para el desarrollo de empresas cooperativas y asociativas en todo el país. ${ }^{8}$

Con base en una denominada alianza estratégica entre el Gobierno de la República, el Ministro de Trabajo y Seguridad Social, el Consejo Nacional de Cooperativas (CONACOOP) y la Universidad Estatal a Distancia (UNED) se renovado la carrera de Administración de Empresas con énfasis en Cooperativas y Asociativas impartida por la UNE y cabe resaltar que es la única en el nivel de Licenciatura ofrecida al Movimiento Cooperativo costarricense y con un alto grado de compromiso de la UNED con este tipo de empresas solidarias, se incorpora un renovado concepto del cooperativismo, con el cual, el desarrollo académico y profesional de los costarricense se ajuste a la realidad del país y a los desafíos de las nuevas teorías del comercio mundial.

La carrera cuenta con cursos específicos en aspectos cooperativos como son el Derecho cooperativo y social, autogestión como modelo de propiedad cooperativa y está especialmente dirigido a cooperativistas, lo cual enriquece a sus participantes, desde nuestro punto de vista, porque les permite relacionarse con sus pares, aprender de sus experiencias y contribuir con sus soluciones dentro de un mismo sector de economía cooperativista y asociativa, indicamos abajo los cursos a que nos referimos y remitimos al lector al anexo III para conocer más fondo los fundamentos, requisitos y cursos de la Carrera.

8 Información obtenida de la pagina Web del periódico Grupo Nación GN, S.A., en columna Vueltaenu dirigida por la periodista Andrea Méndez Montero, anmendez@ vueltaenu.co.cr, bajada el 26 de junio de 2008. 
Derecho Cooperativo y Social

Doctrina Cooperativa y Social

Autogestión y desarrollo Agrario

Desarrollo e Integración Autogestionaria

También existe la Carrera de Técnico Superior en Educación Cooperativa que actualmente capacita a 215 docentes. Esta iniciativa es impulsada por COOPEMEP R.L., CENECOOP R.L., la Universidad Estatal a Distancia (UNED) y el Ministerio de Educación Pública a través del CEE.

\section{Convenios de la Universidad Nacional con el movimiento cooperativo}

Podemos mencionar dos convenios que se ubican dentro de la programación de extensión universitaria y no como programas de carreras dentro de la Universidad. Tales convenios son: el N. 615 denominado Convenio entre la UNA y la Comisión Permanente de Cooperativas de Autogestión. ${ }^{9}$ Y el N. 617, Convenio entre la UNA y el Consejo Nacional de Cooperativas y la FUNA. ${ }^{10}$

\section{Contribución de las Universidades Privadas al desarrollo del quinto principio cooperativo. La labor de la Fundepos-Alma Mater}

Con una experiencia de 25 años la Fundepós Alma Mater ofrece programas de postgrado, de licenciatura y bachillerato en administración de negocios y presta servicios de capacitación y extensión universitaria, así como de asesoría y consultoría a organizaciones de diferente naturaleza. Su objetivo es contribuir a incrementar la capacidad para que se puedan resolver eficientemente los problemas gerenciales de las empresas e institucionales para las cuales ofrece sus servicios a quienes deseen estudiar y obtener su grado de Maestría paralelamente con su desempeño laboral, procurando mejorar a las empresas, a la sociedad y al país en general con sus conocimientos adquiridos. También se orienta a estudiantes de licenciatura y bachillerato universitario, que

${ }^{9}$ Organismo cúpula del movimiento cooperativo asociado de Costa Rica, es parte del Consejo Nacional de Cooperativas.

10 La FUNA es una fundación nacida en el seno de la Universidad Nacional que desarrolla programas de extensión universitaria. 
deseen cursar carreras en el área de las Ciencias Económicas y disciplinas afines. Asimismo, se orienta a ejecutivos de nivel medio y superior de empresas e instituciones, que requieren actualizarse y capacitarse por medio de las acciones de extensión docente que brinda la Universidad Alma Máter.

Es una universidad con participación accionaria de una de las cooperativas de intermediación financiera más fuertes de Costa Rica, COOPEMEX R.L. que tiene dos representantes en su Consejo académico que ocupan actualmente la presidencia y la secretaría ${ }^{11}$. Ofrece diversas carreras en el nivel de bachillerato:

- Contaduría

- Dirección de empresas

En ambas carreras se ofrece en el primer cuatrimestre un curso de Ética profesional.

Cuenta con varios programas de postgrado, como el Programa de Maestría Profesional en Dirección de Empresas con Énfasis en Gestión Capital Humano (M.B.A.)

Es un programa dirigido, básicamente y desde nuestro punto de vista para los administradores de los entes cooperativos, pues tiende a desarrollar las destrezas y profundizar conocimientos en la administración de empresas, al respecto en su promoción la Fundepós-Alma Máter comunica sobre este programa:

«La Universidad Alma Máter, ofrece un prestigioso programa de Maestría Profesional en Dirección de Empresas en Costa Rica, con más de veinte años de experiencia en el desarrollo y administración de maestrías en el área de las Ciencias Económicas, y más de dos mil graduados a la fecha.

Este programa es la mejor opción para actualizar conocimientos y permanecer al día con respecto a las nuevas teorías y técnicas indispensables para triunfar en el ámbito profesional»

Y también ofrece los siguientes programas de maestría:

- Programa de Maestría Profesional en Dirección de Empresas M.B.A.

- Programa de Maestría Profesional en Dirección de Empresas con Énfasis en Banca y Finanzas

- Programa de Maestría Profesional en Dirección de Empresas con Énfasis en Negocios Internacionales.

11 Tomado de la página de la cooperativa COOPEMEX R.L. 25 de junio de 2008 
Si bien, en el contenido de los programas de los postgrados antes mencionados o en las carreras a nivel de bachillerato antes enunciadas, no están contemplados cursos dirigidos al desarrollo de la ideología, doctrina, filosofía del Cooperativismo, la pertenencia de Fundepós al Grupo Empresarial Cooperativo ${ }^{12}$ y la integración de COOPEMEX R.L. en su Consejo Académico define que su mayor mercado se encuentra entre los dirigentes y sobre todo los administradores de las cooperativas. ${ }^{13}$

Cabe destacar que de los requisitos sobresalientes que tiene el Grupo Empresarial Cooperativo y que encontramos cierta relación con los Valores y Principios Cooperativos se encuentran los siguientes:

- Reconocida solvencia moral y económica de la organización. Relacionado con el valor de honestidad.

- Demostrada capacidad de desarrollo empresarial, generación de empleo y compromiso con la comunidad. Relacionado con el sétimo principio.

\section{Programas interuniversitarios relacionados con las cooperativas. Más allá del cooperativismo: la economía social. Red Euro-Latinoamericana de estudios en economía social y cooperativa, (RULESCOOP) del Proyecto ALFA, Unión Europea en Latinoamérica}

RULESCOOP es el resultado de un proyecto ALFA ${ }^{14}$, financiado por la Unión Europea y ejecutado en los años 2003 y 2005. Los miembros de esta red consideraron que su permanencia y crecimiento era importante para estimular la investigación y la docencia con el fin de contribuir desde la actividad académica con el quehacer de las cooperativas y la Economía Social y Solidaria en general.

12 La integración de COOPEMEX R.L. y de FUNDEPOS al Grupo Empresarial Cooperativo y los requisitos de participación pueden ser revisados en el Anexo I.

13 Remitimos al lector al Anexo I para obtener una idea, en forma palpable, de los contenidos de los programas de maestría que hemos mencionado.

14 El Programa ALFA (América Latina Formación Académica), impulsado por la Comisión Europea, se encuentra en su segunda etapa de desarrollo. Tiene como objetivos generales promover la cooperación académica entre universidades de América Latina y Europa, tanto en dimensiones formativas como de desarrollo institucional.

En este marco, el proyecto ALFA «Fortalecimiento de una Red Universitaria Eurolatinoamericana de Estudios Asociativos y Cooperativos», buscaba reunir los esfuerzos colectivos de 5 Universidades de América Latina y 5 universidades de Europa, que históricamente han desarrollo actividades en el campo de la asociatividad, la economía y el cooperativismo. 
El objetivo central de la Red RULESCOOP, tal como se declara en su convenio ínter universitario de cooperación es: «... establecer formas de colaboración en el área de la Economía Social, Economía Solidaria y Cooperativismo, para llevar a cabo actividades docentes, de investigación y de extensión universitaria (divulgación universitaria)».

La Economía Social se constituye en una respuesta a las diversas problemáticas sociales que enfrentan las diferentes sociedades a nivel mundial. Entre las alternativas que la fórmula de la Economía Social ofrece a los diversos colectivos, se rescata la inclusión de grupos tradicionalmente excluidos y el estímulo de micro-emprendimientos, que generan importantes contribuciones al producto interno bruto y a las economías en que estas organizaciones se insertan. De igual forma, el aporte que estas iniciativas realizan a la disminución del problema de desempleo, es uno de los efectos más relevantes en donde se ha tenido éxito.

Otro importante aporte que promueve el sector de la Economía Social es la inclusión y la apertura en la participación a todos los actores sociales. Forman parte de estas poblaciones, grupos étnicos, mujeres, personas con algún tipo de discapacidad, poblaciones en desventaja social, inmigrantes, adultos mayores y jóvenes, quienes han encontrado en las organizaciones de la Economía Social una opción viable para su inclusión en la población económicamente activa.

El escenario en el que se han desarrollado las empresas de la Economía Social, también ha propiciado la innovación con características propias de los principios y valores de estas organizaciones. Es decir, se trata con iniciativas orientadas al fomento de la solidaridad, la igualdad, la equidad y la democracia, sin perder de vista la competitividad empresarial y la necesidad de generar alianzas estratégicas ante la dinámica de la globalización.

Concientes de las necesidad de generar sinergias que promuevan la generación de empleo bajo la fórmula de la Economía Social, basados en el conocimiento de las realidades en la que estas organizaciones se insertan, se llevó a cabo el «I Coloquio Internacional de la Red Euro-Latinoamericana de Estudios y Economía Social y Cooperativa: Los desafíos de la economía social» en Brest, Francia, en mayo de 2006, y organizado por la Universidad de Bretagna Occidental (UBO).

Los resultados de esta actividad fueron muy positivos en el desarrollo de investigaciones y promoción entre los diferentes participantes de las mejores formas de aportar a la sociedad conocimiento acertado y adecuado a las necesidades reales del sector. No obstante, hay mucho por hacer para que este propósito se incorpore cada vez más a la cotidianidad de la interacción entre la academia y el quehacer de la econo- 
mía social. Por esa razón, se promovió el II Coloquio Internacional de RULESCOOP.

Durante el primer Coloquio de RULESCOOP, se tomó el acuerdo de llevar a cabo el II Coloquio Internacional en San José de Costa Rica. Las fechas que se conciliaron fueron 14, 15 y 16 de mayo de 2007, con la Universidad de Costa Rica como sede de

Con los siguientes ejes temáticos:

- La vinculación de la academia con la Economía Social. Cada vez es más importante que la academia se acerque a las actividades que se generan en la Economía Social, Solidaria y de las Cooperativas.

- La responsabilidad social empresarial.

- Políticas públicas dirigidas al fomento del cooperativismo, el asociativismo y la economía social y solidaria, como alternativas para la generación de empleos y la mejora de la calidad de vida de la región euro-americana

\section{Conclusiones}

No hay duda que se ha dado un avance significativo en la educación universitaria dedicada a formar y mejorar a los y las cooperativistas, de la región centroamericana y el Caribe, pues muchas personas de las islas caribeñas se inscriben el Programa de Maestría de administración de empresas cooperativas de la Universidad de Costa Rica. Aunque las verdaderas opciones para la integración educativa de esa región, proviene de entes supranacionales y supraregionales, como son la UniRcoop y la Rulescoop, que más bien integran a profesores e investigadores de temas cooperativos, en interesantes proyectos de investigación-acción, pero que no ofrecen programas de formación de dirigentes o cooperativistas, en el resto de los países de la región antes mencionada, excluida Costa Rica.

La esperanza es que la UniRcoop y Rulescoop se mantengan con vida en el futuro cercano y de largo plazo, y que en sus proyectos se mantenga el interés por impulsar la organización de estudios de licenciatura y postgrado en materia cooperativista, en cada país centroamericano y caribeño.

No hay duda que la $\mathrm{ACl}$ para las Américas ha dado y dará un excelente apoyo al desarrollo de las redes mencionadas, les ha ofrecido su plataforma organizativa en los congresos anuales así como otras instancias de apoyo a sus programas, pero indudablemente y únicamente 
las universidades pueden ofrecer la educación superior y accesible a los cooperativistas, su dirigencia y administradores.

Eso si debemos retomar algunos de los conceptos expresados en el nivel general y planetario por los autores que mencionamos al inicio de este ensayo, cuando nos indicaron por parte del grupo de la Comisión dirigida por J. Delors, la importancia de reconocer la tensión entre la indispensable competencia y la preocupación por la igualdad de oportunidades, porque nos permite elegir entre educación universitaria general para administradores y una dirigida a mantener y valorar la ideología y filosofía cooperativista, en la cual el afán por el respeto al principio de la igualdad sustantiva, es esencial, y en donde muchas organizaciones cooperativas se pueden desviar de su objetivo social, en aras de conseguir altos y continuos ingresos económicos en detrimento del desarrollo y fortalecimiento de las instancias parapolíticas, lugar donde los miembros expresan sus intereses y necesidades en igualdad de posiciones.

Asimismo, tener en cuenta la tensión entre lo espiritual y lo material, que también es una constatación eterna y que principalmente en el ejercicio y la revisión de los efectos de la aplicación extensa y profunda del quinto principio cooperativo, nos hace comprender o recordar que las cooperativas son organizaciones de personas y no de capitales, de tal modo que la bonanza económica debe tener un reflejo real en el bienestar de las personas físicas que las componen. Por eso Edgar Morín en 1999, planteó que la educación puede caer en la ceguera y la ilusión, si no ve al ser humano en toda su extensión, física y espiritual, y la educación ofrece sus productos tomando en cuenta la multiplicidad de perfiles que forman al educando.

Si en la educación cooperativa no se pierde el norte de enseñar la condición humana, las organizaciones cooperativas tendrán dirigentes con excelente preparación académica en la administración de empresas y nunca dejaran el objetivo social que nutre la cooperativa a la que pertenecen, unir academia que da formación y entrenamiento con la educación cooperativa, es el objetivo final de la ejecución del quinto principio cooperativo, cuando hablamos de la relación universidad y cooperativismo.

En el sexto saber, enseñar para la comprensión del ser humano, como ente planetario, será importante revisar la importancia que tiene la coherencia e integración del quinto principio cooperativo, sobre todo cuando hablamos del principio de integración cooperativa y del principio de servicio a la comunidad. Esto porque la integración ya dentro de cada país, a veces resulta difícil y más lo es en una región como la centroamericana, donde las condiciones soció-económicas y políticas 
son dispares y lleva a la lucha hegemónica, de por ejemplo, la ribera de un río como el San Juan; o a reconocer la dura realidad de países donde sus gobernantes de izquierda y derecha se unen en detrimento de la participación de la minorías, como hemos visto recientemente en el país de Nicaragua, lo cual dificulta la integración económica de las cooperativas de los países fronterizos, por considerar la hipótesis mas aceptable.

Frente a esas barreras, sin tomar en cuenta aquellas que surgen con los países insulares del Caribe, por razón también de su diversidad de gobiernos, desarrollo económico y condiciones económicas, la visión de una educación planetaria, se nos hace difícil de aceptar, sino se cambia la mentalidad como indica Morin, para ejercitar la tolerancia, la comprensión en todos los sentidos y que este tipo de aspectos se integren a los contenidos de las pocas ofertas universitarias que existen en la región ce Centroamérica y el Caribe, preparación de líderes, administradores y miembros de las cooperativas. 


\section{Anexo I}

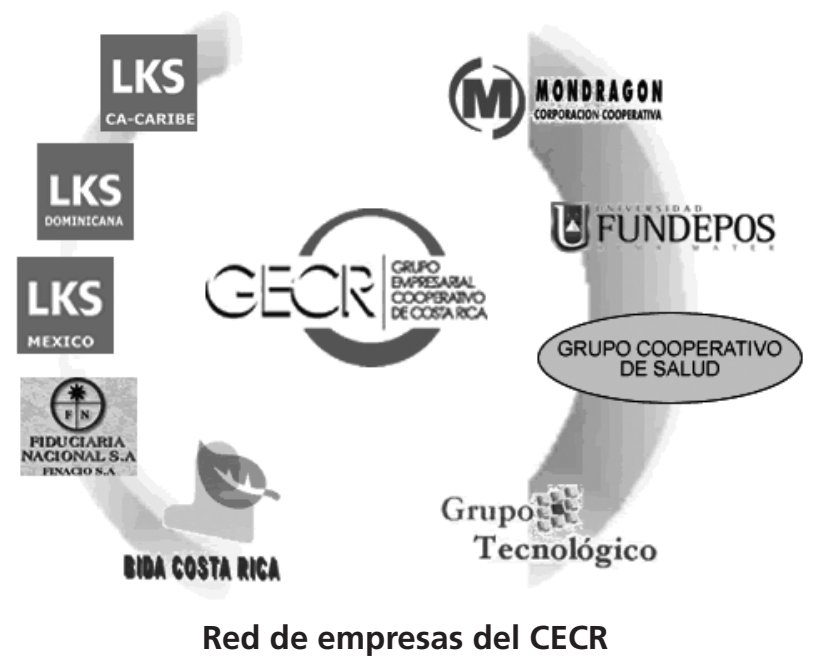

\section{Requisitos de afiliación}

- Suscribir el capital social, establecido por el Consejo de Administración, realizando, el pago del $20 \%$ y el saldo restante en un plazo de 12 meses.

- Aportar al Fondo de Inversiones del GECR un monto, igual al 5\% de sus excedentes netos, en forma anual.

- Cumplir con todos los demás requisitos y obligaciones establecidas en el Estatuto del GECR.

- Contar con trayectoria reconocida dentro del sector de economía social del país.

— Reconocida solvencia moral y económica de la organización.

- Demostrada capacidad de desarrollo empresarial, generación de empleo y compromiso con la comunidad.

—Demostrada capacidad de generar valor al desarrollo del GECR. 


\section{Anexo II}

\section{Programa de maestría profesional en dirección de empresas con énfasis en negocios internacionales (M.B.A.)}

Dirigido a profesionales con grado de Bachillerato universitario (como mínimo) en cualquier disciplina académica, provenientes de universidades debidamente reconocidas por el Consejo Nacional de Rectores (CONARE) o el Consejo Nacional de Educación Privada de Costa Rica (CONESUP)

El programa de Maestría Profesional en Dirección de Empresas con énfasis en Negocios Internacionales, consta de cuatro módulos, a saber: Seminarios de nivelación, cursos Fundamentales, cursos de Énfasis y cursos de Graduación, en éste último módulo se incluye la elaboración del Proyecto Final de Graduación.

\section{Requisitos de admisión}

- Llenar solicitud de admisión al programa.

- Presentar original y fotocopia del título de bachillerato universitario o grado superior. En caso de provenir de una universidad extranjera, presentar el título y el certificado de notas autenticado por el cónsul de Costa Rica en el país donde se realizó el estudio, así como el título ratificado por el Ministerio de Relaciones Exteriores y Culto de Costa Rica.

- Certificación o récord original de notas. Caso de provenir de una universidad extranjera debe cumplir con los mismos requisitos citados en el punto anterior.

- Presentar tres fotos tamaño pasaporte.

- Presentar fotocopia ampliada de la cédula de identidad o pasaporte (por ambos lados).

\section{Módulo I}

Seminarios de nivelación

\begin{tabular}{l|l|l}
\hline Código & \multicolumn{1}{|c|}{ Nombre de curso } & Créd. \\
\hline NIV101 & Nivelatorio de Estadística & n.a. \\
NIV102 & Nivelatorio de Economía & n.a. \\
NIV103 & Nivelatorio de Contabilidad & n.a. \\
NIV104 & Nivelatorio de Finanzas & n.a. \\
\hline
\end{tabular}


Se exime de llevarlos a aquel postulante que haya aprobado, un curso de nivel universitario en cada una de estas áreas. La programación de estos nivelatorios se aplica generalmente bajo la modalidad de tutoría, de manera que la permita al estudiante poder matricular simultáneamente otros cursos del bloque de fundamentales.

\section{Módulo II}

\section{Cursos fundamentales}

Son cursos de nivel analítico, comunes para todos los estudiantes que ingresen al Programa de Maestría Profesional. Estos cursos son los siguientes:

\begin{tabular}{l|l|c}
\hline Código & \multicolumn{1}{|c}{ Nombre de curso } & Créd. \\
\hline FEP200 & Estadística y Negocios & 4 \\
FEP201 & Gerencia de Mercadeo & 4 \\
FEP202 & Economía y Negocios & 4 \\
FEP203 & Contabilidad y Gerencia & 4 \\
FEP204 & Gerencia Financiera & 4 \\
FEP205 & Sist. de Información Gerencial & 4 \\
FEP206 & Globalización Económica & 4 \\
FEP207 & Gerencia y Factor Humano & 4 \\
\hline
\end{tabular}

\section{Módulo III}

\section{Cursos de énfasis}

Son cursos de especialización que profundizan en aspectos relacionados a Gestión Capital Humano, son los siguientes:

\begin{tabular}{c|l|c}
\hline Código & \multicolumn{1}{|c}{ Nombre de curso } & Créd. \\
\hline FEP230 & Desarrollo Económico Global & 4 \\
FEP231 & Comercio y Negocios Internacionales & 4 \\
FEP232 & Medios de Pago Globales & 4 \\
FEP233 & Administración Internacional Comparada & 4 \\
FEP224 & Gerencia Multinacional de Mercadeo & 4 \\
\hline
\end{tabular}




\section{Módulo IV}

\section{Cursos de graduación}

Son el FEP208 Estrategia Empresarial, en este curso se procura integrar los conocimientos adquiridos en el Programa de Maestría y el FEP209 Seminario-Taller, que corresponde a la elaboración del Proyecto Final de Graduación. Para el desarrollo de este último, se brinda un seminario en el cual se desarrollan los principales elementos que debe incluir el Proyecto de graduación. (P.F.G.)

\begin{tabular}{c|c|c}
\hline Código & Nombre de curso & Créd. \\
\hline FEP208 & Estrategia Empresarial & 4 \\
FEP209 & Seminario Taller (P.F.G.) & 8 \\
\hline
\end{tabular}

\section{Módulo IV}

\section{Cursos de graduación}

Son el FEP208 Estrategia Empresarial, en este curso se procura integrar los conocimientos adquiridos en el Programa de Maestría y el FEP209 Seminario-Taller, que corresponde a la elaboración del Proyecto Final de Graduación. Para el desarrollo de este último, se brinda un seminario en el cual se desarrollan los principales elementos que debe incluir el Proyecto de graduación. (P.F.G.)

\begin{tabular}{c|c|c}
\hline Código & Nombre de curso & Créd. \\
\hline FEP208 & Estrategia Empresarial & 4 \\
FEP209 & Seminario Taller (P.F.G.) & 8 \\
\hline
\end{tabular}




\section{Anexo III \\ Universidad Estatal a Distancia}

\section{Plan de Estudio}

Administración de Empresas con énfasis en Empresas Cooperativas y Asociativas

El profesional en el nivel bachillerato, estará en capacidad de dirigir o efectuar el planeamiento y programación, organización, ejecución, supervisión, control y evaluación de la gestión social y empresarial en lo interno y el entorno de la organización.

También estará capacitado para el desarrollo de proyectos productivos que estén contenidos en la democratización de la economía costarricense, para que la población pueda asegurarse la satisfacción de sus necesidades básicas.

\section{Requisitos}

Requisito de ingreso: Tener aprobado el nivel de Bachillerato en Administración de Empresas con Enfásis en Empresas Asociativas y Cooperativas. Aprobación del Encargado de Programa en casos de alumnos con estudios superiores afines que desean ingresar a niveles intermedios

Periodo: Semestre

Grado(s) Académico(s): Diplomado, Bachillerato, Licenciatura

\section{Carreras Similares}

Diplomado

\section{1 er Semestre}

Técnicas de Estudio a Distancia y de Investigación

\section{2..$^{\circ}$ Semestre}

Administración General I

Contabilidad I

Matemática para Administradores I 
3er Semestre

Administración General II

Contabilidad II

Economía General

Matemática para Administradores II

4. ${ }^{\circ}$ Semestre

Administración de Recursos Humanos

Estadística I

Introducción a la Computación

Matemáticas Financieras

5. ${ }^{\circ}$ Semestre

Derecho Empresarial

Estadística II

Finanzas I

Mercadeo Básico

6. ${ }^{\circ}$ Semestre

Historia de La Cultura

Lengua y Literatura

Perspectivas Filosóficas del Hombre

Bachillerato

1 er Semestre

Comportamiento Organizacional

Derecho Cooperativo y Social

Doctrina Cooperativa y Social

Finanzas II

Macroeconomía

Microeconomía

Producción I

2. ${ }^{\circ}$ Semestre

Autogestión y desarrollo Agrario

Estrategia Empresarial I

Operaciones Bursátiles

Planificación Programas Educativos

Preparación y Evaluación de Proyectos

Producción II

3. er Semestre

Electiva Ciclo Complementario

Electiva Ciclo Complementario

Proyecto de Investigación en Administración de Empresas 


\section{Licenciatura}

\section{1. ${ }^{\text {er }}$ Semestre}

Análisis Económico y Político

Estrategia Empresarial II

Finanzas Internacionales

Investigación de Operaciones I

\section{2. ${ }^{\circ}$ Semestre}

Administración del Comercio Internacional

Cambio Organizacional

Desarrollo e Integración Autogestionaria

Sistemas de Información Gerencial

\section{$3^{\text {er }}$ Semestre}

Estrategia para el Cambio

Taller de Investigación Dirigida o Investigación Dirigida

Tendencias Futuras en la Administración

\section{4. ${ }^{\circ}$ Semestre}

Tésis, Proyecto o Seminario

\section{Bibliografía}

Web de la ACl: Documento Informativo N. ${ }^{\circ}$ EHO/101/95 del 17 de noviembre de 1995) (Publicado en «Mundo Cooperativo», N. ${ }^{\circ} 11$, mayo de 1997, Pág. 24, bajado el 20 de junio de 2008.

BRUNo-MARIE Béchard, Rector de la Université de Sherbrooke (Canadá). La Cooperación Como Orden Mundial, discurso con motivo del X Seminario Internacional de la Red universitaria de las Américas en estudios cooperativos y asociativismo (uniRcoop). Copán Ruinas, 2 de noviembre de 2006.

Información obtenida de la pagina Web del periódico Grupo Nación GN, S.A., en columna Vueltaenu dirigida por la periodista Andrea Méndez Montero, anmendez@vueltaenu.co.cr, bajada el 26 de junio de 2008 